InnOvaciOnes de NegOciOs 16(31): 55-86

(c) 2019 UANL, Impreso en México (ISSN: 2007-1191)

Recepción: 21 Noviembre Aceptación: 7 Diciembre 2018

\title{
La auditoría de estados financieros para efectos fiscales como control interno para reducir las contingencias fiscales de las empresas (The financial audit for fiscal pourposes like fiscal internal control as a measure to contribute to the decreasing of fiscal contingencies)
}

\author{
Alfonso Hernández Campos, Vicente Esteban Martínez Pérez, Alba Luna \\ Gómez, Jessica Lizet Hernández Flores \\ Universidad Autónoma de Nuevo León; Instituto Tecnológico de Estudios Superiores \\ de Monterrey \\ alfonsoh@viakable.com; vicentemtza@gmail.com; alba45@hotmail.com;
} A01281123@itesm.mx

\begin{abstract}
The purpose of this article is to assess the advisability of maintaining the audit of financial statements for fiscal purposes (Fiscal Opinion) as part of internal control in the companies for the purpose of reducing the fiscal contingencies that may arise from the exercise of the faculties of verification of the fiscal authorities. In order to carry out the foregoing, the relevant aspects of said audit are analyzed, as well as the main causes that contribute to the continued existence of the large corporate issuer groups in the Mexican Stock Exchange located in the state of Nuevo León, to this end, surveys were applied to both the groups and the firms that audited them, analyzing the results applying the methodology of experts by fuzzy logic. The results indicate that the large groups that continue to pronounce fiscally do so, by virtue of constituting it as an internal control tool that helps eliminate or reduce their fiscal contingencies.
\end{abstract}

Keywords: Financial and Fiscal Audit, Fiscal Internal Control, Fiscal Contingences.

JEL: M41, M42, M.49.

La auditoría de estados financieros 
Resumen. El propósito de este artículo es evaluar la conveniencia de mantener el la auditoría de estados financieros para efectos fiscales (Dictamen Fiscal) como parte del control interno en las empresas para efectos de reducir las contingencias fiscales que pudieran derivarse del ejercicio de las facultades de comprobación de las autoridades fiscales. Para llevar a cabo lo anterior, se analizan los aspectos relevantes de dicha auditoría, así como las principales causas que contribuyen a que se siga manteniendo en los grandes grupos empresariales emisores en la Bolsa Mexicana de Valores ubicados en el estado de Nuevo León, para ello se aplicaron encuestas tanto a los grupos como a las firmas que los auditan, analizando los resultados aplicando la metodología de expertones por lógica borrosa. Los resultados indican que los grandes grupos que continúan dictaminándose fiscalmente lo hacen, en virtud de constituir el mismo como una herramienta de control interno que contribuye a eliminar o reducir sus contingencias fiscales.

Palabras clave: Auditoría Financiera y Fiscal, Control Interno Fiscal, contingencias Fiscales.

\section{Introducción}

El incremento en la participación de las empresas en nuevos mercados globales y domésticos ha traído como consecuencia que el volumen de las operaciones inherentes a sus actividad económica también crezca, este hecho colateralmente incrementa el riesgo de que las mismas pudiesen estar expuestas a regulaciones fiscales locales 0 internacionales que, de no tener bajo su control, pudiesen generar, costos adicionales fiscales, entre ellos destacan los fundamentados en el Código Fiscal de la Federación (CFF), tales como los recargos de conformidad a su artículo 21, las actualizaciones de las contribuciones omitidas según el artículo 23 y las multas inherentes fundamentadas, entre otros, en el

Hernández, A., Martínez, V., Luna A. \& Hernández J. 
artículo 70 del citado ordenamiento jurídico (CFF, 2018); todos estos costos pudiesen afectar en mayor o menor medida las utilidades financieras de los negocios pudiendo incluso complicar su permanencia en el mercado.

Hasta el año de 2013, de conformidad con el artículo 32-A del CFF y el anexo 5 de la primer Resolución Miscelánea Fiscal (RMF) para 2017 (RMF, 2017), las personas morales residentes en México, conjuntamente con la personas físicas con actividades empresariales, que en el ejercicio anterior hubiesen obtenido ingresos acumulables superiores a ciento nueve millones de pesos, o que el valor de su activo haya sido superior u ochenta y siete millones de pesos en los términos de la regla 2.13.13. de la RMF (RMF, 2018), o bien, que por lo menos trescientos de sus trabajadores les hayan prestado servicios en cada de los meses del ejercicio inmediato anterior, se encontraban obligadas a que se les practicara por un auditor independiente, una Auditoría Financiera para Efectos Fiscales también conocida como Dictamen Fiscal (CFF, 2018), adicionalmente a ellos, también podían dictaminarse aquéllos contribuyentes que optaran por hacerlo, pues no estaba limitada esa posibilidad para quien lo deseara; a partir del año de 2014 esa opción desapareció, y solo permite dictaminarse fiscalmente en forma voluntaria a aquellos contribuyentes que cumplieran exclusivamente los requisitos de ingresos, activos o trabajadores citados con antelación (las cantidades de ingresos y activos actualizadas deben ser las vigentes al año a dictaminar); también eliminó la posibilidad de dictaminarse fiscalmente a las entidades paraestales de la Administración Pública Federal.

\section{La auditoría de estados financieros}


El hecho de ser actualmente optativa la opción de dictaminarse fiscalmente, ha puesto en tela de duda la conveniencia de hacerlo principalmente por el costo que representa, por lo cual se vuelve relevante el conocer la opinión de los grandes grupos empresariales que cotizan en la Bolsa Mexicana de Valores (BMV) ubicados en Nuevo León así como de quienes los dictaminan focalizando directamente a las Big Four, respecto a si se continúan dictaminándose fiscalmente y el conocer las principales razones de que lo sigan haciendo.

Un hecho relevante a mencionar es que si bien es cierto se vuelve optativo el dictamen fiscal, el Servicio de Administración Tributaria (SAT) de todas formas sustituyó su información para ciertos contribuyentes, con la Declaración Informativa sobre su situación Fiscal (DISIF) de conformidad al artículo 32-H (CFF, 2018), esta declaración contiene prácticamente la misma información que la reflejada en el dictamen fiscal (Hernández, 2016), solo que no lleva revisión por parte de un auditor externo.

En adición a lo anterior SAT, desde el año de 2014 y con fundamento en los artículos 42 fracción IX y 53B del CFF (CFF, 2018), ha iniciado su fiscalización de manera electrónica con la propia información proporcionada por los contribuyentes ya sea a través de los CFDI 0 Comprobantes Fiscales Digitales regulados principalmente en los artículos 29 y 29-A del CFF, con la contabilidad electrónica (artículo 28 del CFF), con datos proporcionados vía buzón tributario (Artículo 17-K $\mathrm{CFF}$ ), con las diversas declaraciones electrónicas (pagos provisionales, declaración anual, DISIF, declaración country by country, etc.), con la información proporcionada en virtud al acuerdo de intercambio de

Hernández, A., Martínez, V., Luna A. \& Hernández J. 
información fiscal denominado Foreign Account Tax Compliance Act, también conocido por su acrónimo en Inglés como FACTA (IFRS, 2018) celebrado con Estados Unidos, etc.; lo anterior representa sin lugar a dudas retos adicionales en materia del establecimiento de mayores controles internos fiscal en las empresas (Hernández, 2016), en virtud a que esta información el SAT prácticamente la tiene en línea por lo que en sus revisiones electrónicas, podrá cruzar en forma inmediata la información recibida y, en su caso, emitir pre liquidaciones por omisión de contribuciones, detectar oportunamente estrategias fiscales agresivas, mejorar el intercambio de información con otras jurisdicciones (países) y supervisar el cumplimiento de las obligaciones fiscales que tiene dadas de alta el contribuyente (PWC, 2016).

Por lo anterior, se vuelve relevante que las compañías focalicen sus esfuerzos en sacar mayor provecho a las tecnologías de información, tal como lo está haciendo el SAT según lo revela su segundo informe de gestión de 2018, en el que se observan actos de fiscalización a través de Buzón tributario, requerimientos, correos electrónicos, etc. (SAT, 2018). El problema se intensifica en las empresas que dejan de tener la auditoría fiscal pues esta solía ser un medio de apoyo para validar y/o corregir oportunamente sus operaciones fiscales; para con ello reducir el riesgo de incurrir en contingencias fiscales. 
60

\section{Marco Teórico.}

\section{Generalidades}

En un entorno altamente competitivo, las empresas buscan continuamente la optimización de sus recursos con la finalidad de subsistir en el mercado en que participan, lo anterior se logra al ser eficaz, pues ello implica el alcanzar sus metas (Robbins \& Coulter, 2000) de forma eficiente, lo que conlleva al adecuado manejo de los recursos con los que cuenta; todo esto se materializa en alcanzar el objetivo primordial para el que fueron creadas: la generación de utilidades financieras mismas que les permite mantenerse como negocio en marcha de conformidad en apego a las Normas de Información Financiera (CINIF, 2018).

La generación de mayores utilidades de operación, y consecuentemente de mayores utilidades financieras en los negocios, principalmente implica el incrementar las ventas de los productos 0 servicios con los mayores márgenes de utilidad posibles o bien, la reducción de sus costos de operación.

El incrementar los niveles de ventas suele ser un verdadero reto, tomando en cuenta la posible existencia de un mercado altamente competitivo que suele tener las mismas aspiraciones, por ello es muy importante que se cuenten con diferenciadores que agreguen valor a sus productos o servicios y sea apreciado por sus clientes para que se materialice en la venta; en la medida que los diferenciadores sean relevantes contribuirían a prácticamente no competir pues estaría

Hernández, A., Martínez, V., Luna A. \& Hernández J. 
posicionado en un nuevo mercado sin competidores (Chan \& Mouborgne, 2005).

En relación a la reducción de costos, si bien es cierto, conceptualmente pareciera ser una buena alternativa para incrementar los márgenes de utilidad, se deben considerar todas las posibles repercusiones que se pudieran tener por tomar esa decisión. El caso de la Auditoría Financiera para Efectos Fiscales también conocida en México como Auditoría Fiscal, constituye uno de esos casos que generan polémica en su utilización, en virtud a que el costo de dictaminarse se repercute al contribuyente que decide utilizarlo (Decreto, 2010); es por ello que la decisión de dejarlo de utilizar se debe realizar a través de un análisis integral pero partiendo de las particularidades propias de cada empresa, sobre todo por el hecho de que la evidencia científica señala que las empresas grandes y medianas no cuentan con suficientes controles internos fiscales que garanticen el adecuado cumplimiento de sus disposiciones fiscales, aunado al hecho de que desde la perspectiva industrial y de las Big Four, no existe suficiente personal fiscal para controlar fiscalmente todas las operaciones (Hernández, 2011).

Auditoría financiera

Las operaciones comerciales realizadas por las empresas con sus clientes o proveedores implican regularmente actos de comercio de conformidad al artículo 75 del Código de Comercio (CC) (CC, 2018), sin embargo, la garantía de recibirlos lleva implícitamente un riesgo inherente en el sentido de que realmente suele desconocerse si el proveedor 0 cliente cuenta con la capacidad necesaria para llevar a cabo esas

\section{La auditoría de estados financieros}


operaciones, es por ello que la auditoría financiera contribuye a validar que la empresa al menos con los elementos necesarios para continuar operando, y consecuentemente, que las transacciones se lleven a cabo.

Lo anterior es relevante si tomamos en cuenta que en México la mayoría de las empresas se constituyen como Sociedades de Responsabilidad Limitada o como Sociedades Anónimas, las cuales solo responden ante terceros a través del monto de sus aportaciones de conformidad al artículo 58 y 87 de la Ley General de Sociedades Mercantiles (LGSM), es importante señalar que derivado de la reforma de 2002 ya no se requiere un capital mínimo para constituirse (LGSM, 2018).

Por lo anterior, se vuelve relevante que se lleve a cabo la auditoría financiera, no obstante ha mermado su credibilidad principalmente por escándalos financieros alrededor del mundo (Largay, 2002), siendo el más sobresaliente el que ocurrió en los Estados Unidos de América (EUA) en el que inclusive ocasionó la desaparición de Artur Andersen como una de las firmas de auditores más importantes pasando de ser las Big Five a las Big Four por la quiebra de Enron (PWC). En contramedida a los escándalos financieros en los EUA, surge la Ley Sarbanes Oxley también conocida como SOX (Fernández, Vera y Silvia, 2015), en ella pone especial interés en la actuación y responsabilidad del auditor externo respeto a las empresas que cotizan en la bolsa de dicho país, particularmente en la sección 303 enfatiza sobre el cumplimiento de las leyes y el control interno. La Ley SOX goza de extraterritorialidad pues se tiene que cumplir independientemente de dónde se encuentren ubicadas, pues el hecho generador de la obligación es el cotizar en la Bolsa de Nueva York (Carpi, 2010).

Hernández, A., Martínez, V., Luna A. \& Hernández J. 
En virtud a que el Dictamen fiscal lleva consigo la práctica de una auditoría financiera para efectos fiscales, se hace necesario partir tanto del concepto de la Auditoría Financiera, así como de los efectos fiscales medidos en función a los Impuestos Federales existentes en México. En primer lugar el concepto de Auditoría Financiera se puede definir como aquella por medio de la cual se lleva a cabo la revisión de información contenida en los Estados Financieros (Estado de Situación Financiera, Estado de Resultado Integral, Estado de Cambios en el Capital Contable y Estado de Flujos de Efectivo) y las partidas que los integran por parte de un auditor competente (Contador Público Certificado) con los conocimientos y experiencia necesaria para emitir una opinión sobre la razonabilidad de los mismos en materia del cumplimiento a las disposiciones normativas financieras vigentes $y$ aplicables a la fecha de su revisión.

Las normas financieras se reglamentan en las NIF (Normas de Información Financiera) emitidas por el Consejo Mexicano de Normas de Información Financiera conocido como CINIF (CINIF, 2018) o bien en las Normas internacionales de Información Financiera (NIIF) también conocidas como IFRS por su acrónimo en inglés (International Financial Reporting Standard) emitidas por la Fundación de IFRS y por the International Accounting Standards Board (IASB) (IFRS, 2018) las cuales son obligatorias de adoptar en sus Estados financieros para aquellos grupos de empresas que cotizan en la Bolsa Mexicana de Valores (BMV) (SHCP, 2009).

Para estar en condiciones de emitir una opinión, la revisión del auditor requiere de la aplicación de diferentes pruebas de auditoría las

\section{La auditoría de estados financieros}


cuales normalmente están en función a la naturaleza propia de cada empresa a auditar, pero sobre todo en existencia y eficacia de los controles internos que tiene implementados; por cumplimiento normativo, no hay disposición que obligue a tener controles internos (Hernández, 2016), sin embargo por normativa extranjera para las empresas que cotizan en la Bolsa de Valores de Nueva York, se requiere su validación por parte del auditor (Carpi, 2010). A partir de 2012 en México, paralelamente con la entrada en vigor de las IFRS para las empresas de la BMV, es obligatoria la aplicación de las Normas Internacionales de Auditoría (SHCP, 2009), en ellas contemplan dos tipos de opinión, la No Modificada, antes conocida como opinión limpia y la Modificada la cual anteriormente se dividía en tres categorías: opinión negativa, con salvedades o con abstención de opinión.

Las únicas empresas o grupos de empresas que están obligadas a dictaminarse para efectos financieros son aquellas que se encuentran en la Bolsa Mexicana de Valores, lo anterior encuentra fundamento en el artículo 85 fracción III y artículo 87 fracción I de la Ley de Mercado de Valores (LMV), es importante señalar que el auditor responderá ante los daños y perjuicios que ocasione en caso de que incurra en algún supuesto que contempla el artículo 370 de la citada Ley, los cuales pueden ser el omitir información relevante, presentar información falsa, recomienden operaciones contrarias a los principios de contabilidad, etc. (LMV, 2018).

En la actualidad, son 145 Grupos de empresas que cotizan en la BMV, cabe señalar que existen algunas empresas adicionales que si bien es cierto no cotizan en la Bolsa, también reportan información financiera

Hernández, A., Martínez, V., Luna A. \& Hernández J. 
al emitir obligaciones de forma pública, las cuales también deben de proporcionar estados financieros dictaminados por un auditor externo independiente (SHCP, 2009).

Las principales firmas de contadores que proporcionan servicios de auditoría externa en el mundo son las denominadas Cuatro Grandes mayormente conocidas por su denominación en inglés como Big Four, las cuales son Deloitte, Price Waterhouse Coopers (PWC), Klynveld, Peat, Marwick \& Goerdeler Company (KPMG) y Ernst \& Young (E\&Y), estas firmas son las que dominan la prestación de servicios de auditoría financiera en México respecto a las empresas emisoras, esto es congruente con el estudio realizado en Nueva Zelanda en donde se encontró que a mayor tamaño de las empresas seleccionan a firmas con mayor reputación pudiendo ser las Big Five existentes hasta 2002 (Hay, 2002). Derivado del análisis de los grupos emisores existentes en la BMV, la distribución de las firmas que prestan los servicios de auditoría financiera en México, se refleja en la figura 1 siguiente:

Figura 1. Distribución de Auditorías Financieras respecto a emisoras de la BMV

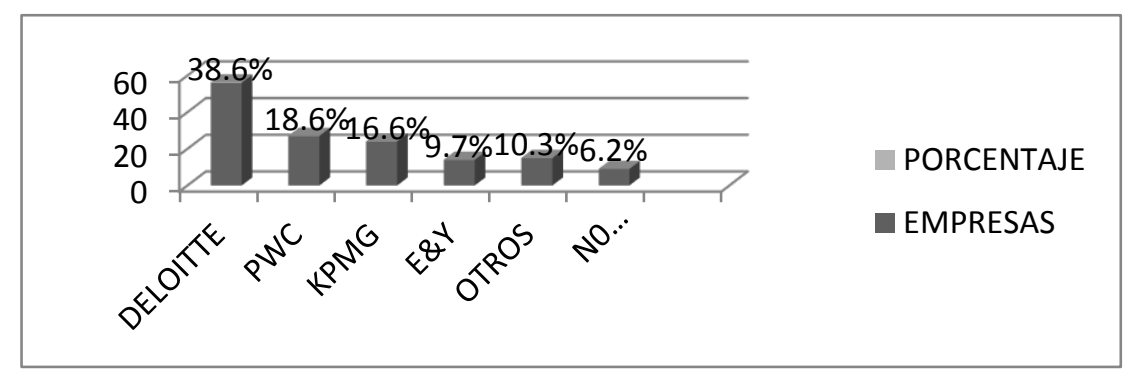

Fuente: Elaboración propia con información públicas de la BMV 2018.

La auditoría de estados financieros 
De la figura 1, se desprende que la Big Four que domina la prestación de los servicios de auditoría financiera en México es Deloitte, seguida por PWC, después KPMG y por último $E \& Y$, es importante aclarar que del análisis de la información de la BMV hubo 9 empresas de las cuales no se localizó información pública al respecto y el restante $10.3 \%$ corresponde otras firmas de contadores no catalogadas como Big Four que también prestan servicios de auditoría financiera. Es relevante señalar que la información se obtuvo del análisis de los estados financieros publicados en la BMV para empresas o grupos de emisoras durante el año de 2018, los cuales podrían corresponder a los ejercicios financieros de 2016 y 2017 en virtud a que no en todos los casos se encontraron los Estados Financieros del ejercicios de 2017.

Del análisis de la información pública del ejercicio de 2017, respecto a las emisoras según la BMV, Deloitte audita la mayor parte del sector Industrial, el de materiales, el correspondiente a productos de consumo frecuente, el de servicios financieros y el de energía. KPMG y PWC dominan, con la misma cantidad de grupos o compañías auditadas, el sector de servicios y bienes de consumo no básicos; por su parte E\&Y, domina el sector de las tecnologías de información y PWC domina el sector de los servicios de telecomunicaciones; respecto al sector salud, no hay un claro dominante pues tienen las mismas compañías PWC, KPMG, Deloitte y Moore Stephens.

Como se comentó con antelación, como resultado de la auditoría financiera, el dictaminador emite una opinión sobre el cumplimiento de la normatividad aplicable, en este caso, por ser emisoras de la BMV, se aplica en la revisión de estados financieros el adecuado apego a las IFRS;

Hernández, A., Martínez, V., Luna A. \& Hernández J. 
por otra parte es relevante mencionar que como parte de la auditoría se hacen análisis a las partidas que tienen que ver con los impuestos federales atendiendo las NIA, por lo tanto, el resultado de sus pruebas y su impacto en el tipo de opinión, en el caso de que existan desviaciones a la normatividad, va en función sobre la importancia de las mismas respecto a los estados financieros en su conjunto, esto se conoce como materialidad o importancia relativa, lo anterior de conformidad con la NIA 320 emitida por el International Auditing and Assurance Standards Board (IAASB, 2009). Los resultados del análisis a las emisoras respecto a las opiniones que se reflejaron en los dictámenes financieros emitidos respecto al ejercicio 2016 y/o 2017 se muestran en la figura 2 siguiente:

Figura 2. Tipos de Opinión de Dictámenes Financieros de emisoras de la $B M V$

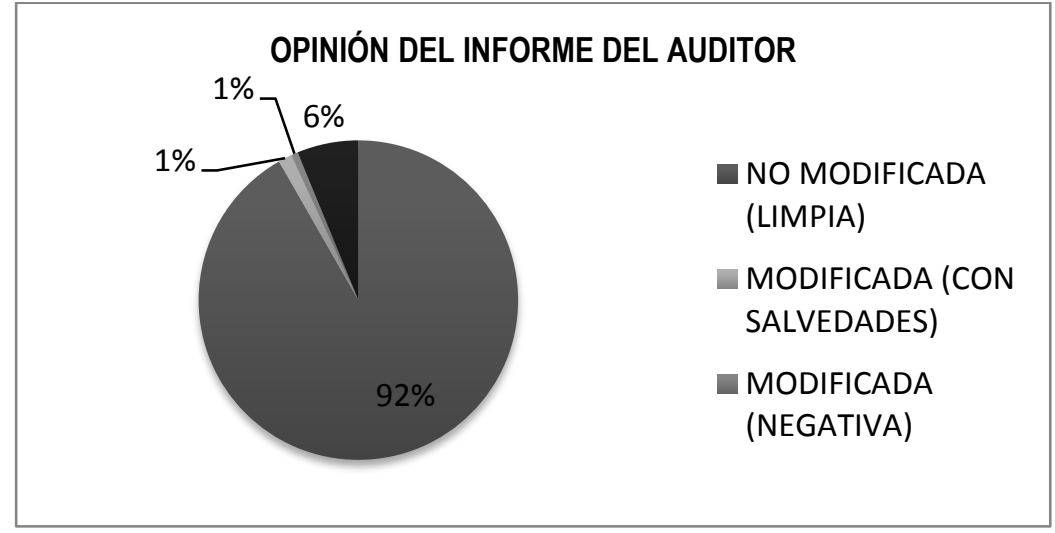

Fuente: Elaboración propia con datos públicos de la BMV 2018.

La auditoría de estados financieros 
De la figura que antecede se desprende que la mayoría de las opiniones del auditor emiten una opinión no modificada con el $92 \%$, es decir que los Estados financieros muestran de manera razonable la información de conformidad a la normatividad aplicable, solo el $1 \%$ fue opinión negativa, es decir que no representa razonablemente la información financiera y otro 1\% fue una opinión con salvedades, es decir que la mayor parte de la información fue razonablemente correcta con excepción de alguna(s) cuenta(s) que no lo son aunque no fue suficiente para que fuera una opinión negativa ni limpia. Cabe señalar que el $6 \%$ de las empresas emisoras no se encontró información. Por otra parte, de la información de la BMV respecto a las emisoras se pudo detectar, mediante el análisis de las notas a los Estados Financieros, que el 35\% de las empresas o grupos cuentan con el Dictamen Fiscal o con algún tipo de revisión fiscal externa, el 59\% no se encontró información que implicara que se dictaminara fiscalmente y del restante $9 \%$ de las emisoras simplemente no se encontró información. En adición a lo anterior se encontró que el $31 \%$ de las emisoras cuentan con algún tipo de contingencia fiscal, el $63 \%$ no cuentan con ella y del $6 \%$ restante no se encontró información al respecto.

Un aspecto interesante a comentar el hecho de que las emisoras de la BMV se encuentran localizadas en diferentes lugares, en la tabla 1 siguiente se muestra su distribución:

Hernández, A., Martínez, V., Luna A. \& Hernández J. 
Tabla 1. Relación de empresas distribuidas en función a su domicilio

\begin{tabular}{lll}
\hline ESTADO & EMPRESAS & $\%$ \\
\hline CDMX & 95 & $65.52 \%$ \\
NUEVO LEÓN & 20 & $13.79 \%$ \\
INFORMACIÓN NO DISPONIBLE & 9 & $6.21 \%$ \\
JALISCO & 6 & $4.14 \%$ \\
CHIHUAHUA & 4 & $2.76 \%$ \\
\hline COAHUILA & 2 & $1.38 \%$ \\
GUANAJUATO & 2 & $1.38 \%$ \\
BAJA CALIFORNIA & 1 & $0.69 \%$ \\
ESTADO DE MÉXICO & 1 & $0.69 \%$ \\
SINALOA & 1 & $0.69 \%$ \\
QUINTANA ROO & 1 & $0.69 \%$ \\
CULIACAN & 1 & $0.69 \%$ \\
DURANGO & 1 & $0.69 \%$ \\
EXTRANJERO (ESPANA) & 1 & $0.69 \%$ \\
\hline TOTAL & 145 & $\mathbf{1 0 0 . 0 0 \%}$ \\
\hline
\end{tabular}

Fuente: Elaboración propia con datos de la BMV consultados en 2018.

Como puede observarse de la tabla 1 que antecede, la mayor concentración de las empresas o grupos auditados corresponde a la Ciudad de México (CDMX) con 95, esto cual se puede explicar por ser el principal asiento para realizar operaciones comerciales en México, aunque no necesariamente implique que su operación se concentre en ese lugar, en segundo lugar se encuentra Nuevo León con 20 empresas emisoras seguido de 25 emisoras que están distribuidas en diferentes México y una se encuentra domiciliada en España.

\section{La auditoría de estados financieros}




\section{Dictamen Fiscal}

Para efectos de tener mayor claridad respecto al origen dictamen fiscal es necesario partir de la obligación constitucional de los mexicanos al contribuir al gasto público fundamentada en el artículo 31 fracción IV de la Constitución Política de los Estados Unidos Mexicanos (CPEUM), es por ello que las empresas, como personas morales, que se encuentren en la situación jurídica o de hecho prevista por las leyes fiscales tienen la obligación de contribuir al gasto público de la manera proporcional y equitativa que las mismas dispongan (CPEUM, 2018). En México, los principales impuestos federales, en función a al presupuesto de ingresos para el ejercicio de 2017, son el Impuesto Sobre la Renta (ISR), seguido por el Impuesto al Valor Agregado (IVA) y el Impuesto Especial sobre Producción y Servicios (IEPS) (CEFP, 2017 p.13), para 2018 y 2019, ese mismo orden de importancia se mantiene en los presupuestos de ingresos de 2018 y 2019 de conformidad en la tabla 2:

Tabla 2. Comparativo de bases históricas de impuestos en miles de millones de pesos

\begin{tabular}{|c|c|c|c|c|c|c|c|c|c|c|c|c|c|c|}
\hline CONCEPTO & 2013 & $\%$ & 2014 & $\%$ & 2015 & $\%$ & 2016 & $\%$ & 2017 & $\%$ & 2018 & $\%$ & 2019 & $\%$ \\
\hline & $\$ 818$ & 51 & $\$ 1,0$ & 0 & $\$ 1,0$ & 0 & $\$ 1,2$ & 0 & $\$ 1,4$ & 52 & $\$ 1,5$ & 53 & $\$ 1,7$ & 53 \\
\hline ISR & .1 & $\%$ & 06.4 & $\%$ & 59.2 & $\%$ & 49.3 & $\%$ & 25.8 & $\%$ & 66.2 & $\%$ & 52.5 & $\%$ \\
\hline IETU & 44.6 & $\begin{array}{l}\% \\
0\end{array}$ & 0.0 & $\begin{array}{l}\% \\
0\end{array}$ & 0.0 & $\begin{array}{l}\% \\
0\end{array}$ & 0.0 & $\begin{array}{l}\% \\
0\end{array}$ & 0.0 & $0 \%$ & 0.0 & $0 \%$ & 0.0 & $0 \%$ \\
\hline IDE & $\begin{array}{l}3.9 \\
62 ?\end{array}$ & $\begin{array}{l}\% \\
39\end{array}$ & $\begin{array}{l}0.0 \\
609\end{array}$ & $\%$ & $\begin{array}{l}0.0 \\
703\end{array}$ & $\%$ & $\begin{array}{l}0.0 \\
742\end{array}$ & $\begin{array}{l}\% \\
0\end{array}$ & $\begin{array}{l}0.0 \\
797\end{array}$ & $\begin{array}{l}0 \% \\
29\end{array}$ & $\begin{array}{l}0.0 \\
876\end{array}$ & $\begin{array}{l}0 \% \\
30\end{array}$ & $\begin{array}{l}0.0 \\
995\end{array}$ & $\begin{array}{l}0 \% \\
30\end{array}$ \\
\hline IVA & 6 & $\begin{array}{l}\% \\
3\end{array}$ & $\begin{array}{l}4 \\
134 .\end{array}$ & $\begin{array}{l}\% \\
0\end{array}$ & $\begin{array}{l}8 \\
160 .\end{array}$ & $\begin{array}{l}\% \\
0\end{array}$ & $\begin{array}{l}0 \\
348 .\end{array}$ & $\begin{array}{l}\% \\
0\end{array}$ & $\begin{array}{l}7 \\
433 .\end{array}$ & $\begin{array}{l}\% \\
16\end{array}$ & $\begin{array}{l}9 \\
421 .\end{array}$ & $\begin{array}{l}\% \\
14\end{array}$ & $\begin{array}{l}2 \\
437 .\end{array}$ & $\begin{array}{l}\% \\
13\end{array}$ \\
\hline IEPS & 53.0 & $\%$ & 4 & $\%$ & 0 & $\%$ & 9 & $\%$ & 9 & $\%$ & 8 & $\%$ & 9 & $\%$ \\
\hline
\end{tabular}

Hernández, A., Martínez, V., Luna A. \& Hernández J. 


\begin{tabular}{|c|c|c|c|c|c|c|c|c|c|c|c|c|c|c|}
\hline & & 0 & & 0 & & 0 & & 0 & & & & & & \\
\hline ISAN & 6.1 & $\begin{array}{l}\% \\
2\end{array}$ & 6.7 & $\begin{array}{l}\% \\
0\end{array}$ & 6.6 & $\begin{array}{l}\% \\
0\end{array}$ & 7.3 & $\begin{array}{l}\% \\
0\end{array}$ & 8.7 & $0 \%$ & 10.6 & $0 \%$ & 10.7 & $0 \%$ \\
\hline ICOMEX & 28.1 & $\begin{array}{l}\% \\
2\end{array}$ & 26.8 & $\begin{array}{l}\% \\
0\end{array}$ & 27.9 & $\begin{array}{l}\% \\
0\end{array}$ & 36.3 & $\begin{array}{l}\% \\
0\end{array}$ & 45.8 & $2 \%$ & 47.3 & $2 \%$ & 70.3 & $2 \%$ \\
\hline OTROS & 28.8 & $\%$ & -13.5 & $\%$ & 21.4 & $\%$ & 23.9 & $\%$ & 27.5 & $1 \%$ & 34.6 & $1 \%$ & 44.7 & $1 \%$ \\
\hline TOTAL DE & $\$ 1,6$ & 97 & $\$ 1,7$ & 0 & $\$ 1,9$ & 0 & $\$ 2,4$ & 0 & $\$ 2,7$ & 10 & $\$ 2,9$ & 10 & $\$ 3,3$ & 10 \\
\hline IMPUESTOS & 05.2 & $\%$ & 70.2 & $\%$ & 79.0 & $\%$ & 07.7 & $\%$ & 39.4 & $0 \%$ & 57.5 & $0 \%$ & 11.4 & $0 \%$ \\
\hline $\begin{array}{l}\text { \% S/IT } \\
\text { INGRESOS }\end{array}$ & $41 \%$ & & $40 \%$ & & $42 \%$ & & $51 \%$ & & $56 \%$ & & $56 \%$ & & $57 \%$ & \\
\hline TOTALES & $\$ 3,9$ & 97 & $\$ 4,4$ & 0 & $\$ 4,6$ & 0 & $\$ 4,7$ & 0 & $\$ 4,8$ & 10 & $\$ 5,2$ & 10 & $\$ 5,8$ & 10 \\
\hline & 56.4 & $\%$ & 67.2 & $\%$ & 94.7 & $\%$ & 63.9 & $\%$ & 88.9 & $0 \%$ & 79.7 & $0 \%$ & 38.1 & $0 \%$ \\
\hline & & & & & & & & & & & & & & \\
\hline $\begin{array}{l}\text { INCREMENT } \\
0\end{array}$ & & & $\begin{array}{l}12.9 \\
1 \%\end{array}$ & & $\begin{array}{l}5.09 \\
\%\end{array}$ & & $\begin{array}{l}1.47 \\
\%\end{array}$ & & $\begin{array}{l}2.62 \\
\%\end{array}$ & & $\begin{array}{l}7.99 \\
\%\end{array}$ & & $\begin{array}{l}11.4 \\
2 \%\end{array}$ & \\
\hline
\end{tabular}

Fuente: Elaboración propia con datos publicados en el DOF

De la tabla que antecede, se puede observar una tendencia hacia la alza en materia de recaudación del 2015 a la fecha, ésta se enfatiza para el año 2019 , en el que se espera recibir un $11.42 \%$ más respecto al ejercicio de 2018, este incremento porcentual se sustenta principalmente en la estrategia del nuevo gobierno para incrementar la recaudación en ISR e IVA es por mayor eficiencia recaudatoria en materia de emisión de comprobantes fiscales digitales por Internet (CEFP, 2019). Por lo anterior los contribuyentes deben considerar como parte de su estrategia de negocios el cuidado en la determinación y entero de las contribuciones federales a los que se encuentra sujeto; el Dictamen Fiscal constituye una herramienta útil para lograrlo, pues en sus anexo información relevante de dichos impuestos, los cuales la autoridad fiscal espera que se estén aplicando en las compañías. El SAT utiliza el sistema de

\section{La auditoría de estados financieros}


presentación del dictamen conocido como SIPRED, cuyos anexos se describen brevemente en la tabla 3:

\section{Tabla 3: Relación de anexos del dictamen fiscal 2017}

\begin{tabular}{l} 
Concepto \\
\hline Datos de Identificación del \\
Contribuyente, del Contador Público, \\
del Representante Legal y Datos \\
Generales. \\
Anexos del 1 al 4 . Son los Estados \\
Básicos: Situación Financiera, \\
Resultado Integral, de Cambios en el \\
Capital Contable y de Flujos de \\
Efectivo. El anexo 4.1 se refiere a las \\
notas de los Estados Financieros (EF). \\
Anexo 5 . Integración analítica de \\
ventas o ingresos netos. \\
Anexo 6 . Determinación del costo de lo \\
vendido para efectos contables y del \\
impuesto sobre la renta.
\end{tabular}

Anexo 7. Análisis comparativo de las subcuentas de gastos.

Anexo 8. Análisis comparativo de las subcuentas del resultado integral de financiamiento.

Anexo 9. Relación de contribuciones a cargo del contribuyente como sujeto directo 0 en su carácter de retenedor. Anexo 10. Relación de contribuciones por pagar.

\section{Descripción Breve de los anexos}

En estos apartados se refleja la información necesaria para evidenciar la responsabilidad de quienes firman respecto a la compañía objeto de la auditoría fiscal y se incluyen datos generales de los involucrados.

En estos anexos se reflejan los saldos finales objeto de la auditoría fiscal que se le practicó por parte del Contador Público Registrado; es importante señalar que esta información debe serla misma a la señalada en otros anexos.

Muestra los diferentes tipos de ingresos, su importancia en \%, variación anual y explicaciones y si fue acumulable para ISR.

Al considerarse como una de las principales deducciones fiscales en las empresas industriales se concilian sus elementos para efectos contables y fiscales.

Se desglosan por subcuenta los gastos de fabricación, venta y administración en los que incurrió el contribuyente identificando tanto los deducibles como no deducibles para ISR explicando las principales variaciones respecto al año anterior.

Muestra el detalle de los intereses, fluctuaciones cambiarias y se categorización como ingresos acumulables o no y como gastos deducibles o no para efectos del ISR.

Este anexo concentra prácticamente el resultado de la auditoría fiscal en materia de las contribuciones a cargo del contribuyente, muestra en su caso las diferencias de impuestos detectadas. Incluye el anexo 9.1 en la que el representante legal manifiesta bajo protesta de decir verdad que se incluyen todas las contribuciones federales de su representada.

Incluye las diferencias a cargo al cierre del año y la información relativa s su pago.

\section{Hernández, A., Martínez, V., Luna A. \& Hernández J.}


Anexo 11. Conciliación entre el resultado contable y fiscal para efectos del Impuesto Sobre la Renta Anexo 12 al 14 señala Operaciones financieras derivadas contratadas con residentes en el extranjero, inversiones permanentes y accionistas.

Anexo 15. Conciliación de ingresos según el Estado de Resultados Integral, el ISR e IVA.

Anexos 16 y 17 . Operaciones e información con Partes Relacionadas (PR).

Anexo 18. Datos Informativos.

Anexo 19. Información de Pagos de ISR e Impuesto al activo por Desconsolidación fiscal.

Anexo 20. Inversiones

Anexo 21. Cuentas y Documentos por pagar en moneda extranjera.

Anexo 22. Préstamos del Extranjero.

Anexo 23. Integración de Pérdidas

Fiscales de ejercicios Anteriores.

Cuestionario de Diagnóstico Fiscal

(Revisión del Contador Público)

Cuestionario en materia de

cumplimiento de Precios de

Transferencia (Revisión del Contador Público)
Se determina el resultado fiscal partiendo del resultado contable desglosando las partidas con connotaciones fiscales y contables.

Se detallan las operaciones financieras para efectos de tener el dato puntual de estas operaciones que normalmente representan deducciones fiscales en empresas mexicanas.

Detalla los tipos de ingresos e identifica cuáles de ellos se consideran para efectos del ISR e IVA.

Muestra las operaciones con PR que deben de estar soportadas con el método de Precio de Transferencia (PT) aplicado.

Muestra datos relevantes tales como Coeficiente de Utilidad, Cuenta de Utilidad Fiscal Neta, etc.

Aplica para aquellos grupos de empresas que optaron por consolidar su información fiscal.

Detalla las inversiones, altas y bajas para ISR.

Incluye los nombres, saldos, tipo de moneda y si es Parte Relacionada o no.

Detalla los préstamos y las retenciones de ISR.

Muestra el origen por año de cada pérdida fiscal generada actualizada y su remanente.

Muestra una serie de cuestionamientos relacionados con la revisión del auditor respecto a los controles internos y demás impuestos a que está sujeto el contribuyente.

Revela, a través de cuestionamientos, la revisión del auditor en materia de PT y su apego a las disposiciones fiscales.

Fuente: Elaboración propia con datos de anexos del Dictamen 2017

En la tabla que anterior se muestra una gran cantidad de información tanto financiera como fiscal, la cual es objeto de revisión por

\section{La auditoría de estados financieros}


parte del auditor fiscal y su equipo de trabajo, los cuales cuentan con la capacitación necesaria para estar en condiciones de emitir una opinión al respecto; aunado al hecho de que deben contar con autorización por parte del SAT para hacerlo. Los Contadores Públicos Registrados (CPR) se pueden verificar en la página oficial del SAT siguiente: https://www.consulta.sat.gob.mx/cprsinternet/, vale la pena señalar que los CPR están capacitados pues es requisito para dictaminar de conformidad al artículo 52 fracción I del CFF (CFF, 2018). En virtud a que las empresas difícilmente cuentan con una estructura fiscal para el manejo de esas operaciones (Hernández, 2011) aunado al hecho de que difícilmente tendrían la misma capacitación que un CPR, se incrementa el riesgo de no estar cumpliendo con las disposiciones fiscales, lo que deriva en la posibilidad de incurrir en multas, actualizaciones y recargos en el caso de que sean observados por la autoridad fiscal, los cuales suelen ser considerables si tomamos en cuenta que la autoridad tiene según el artículo 67 del CFF 5 años para llevar a cabo sus facultades de comprobación aunado al hecho de que exige que los impuestos omitidos sean actualizados por la inflación y se paguen recargos por concepto de intereses a favor del fisco los cuales por citar un año, en 2018 fueron del orden del $1.3 \%$ mensual; además de ellos, dependiendo del tipo de omisión, hay multas que pueden rondar hasta porcentajes mayores el $100 \%$ de la contribución omitida. Los controles internos fiscales son una contramedida que contribuye para garantizar el adecuado cumplimiento de las disposiciones fiscales (Hernández, 2016), sin embargo, las disposiciones fiscales en México están en constante actualización a través de cambios de las propias leyes tributarias, las Resoluciones

Hernández, A., Martínez, V., Luna A. \& Hernández J. 
Misceláneas Fiscales, Los criterios no vinculativos del SAT, etc., es por ello que difícilmente los contribuyente tengan al día esos controles, por lo que el ejercer la opción de dictaminarse voluntariamente para efectos fiscales puede convertirse un buen control preventivo, en virtud a la cantidad de información que tiene que validar el auditor.

El dictamen fiscal surge en el año de 1959 y su obligatoriedad fue en función a que constituía un medio para facilitar a la autoridad hacendaria su fiscalización así como otorgar certeza a los contribuyentes en el cumplimiento de las disposiciones fiscales (IMCP, 2013), sin embargo con la reforma 2014, y a propuesta del presidente en turno, iba a eliminarse totalmente 2014 (E\&Y, 2013), sin embargo, quedó de manera optativa para ciertos contribuyentes tal como se comentó con antelación. El resultado de la reforma al CFF fue el eliminar la obligación de dictaminarse fiscalmente para la mayoría de los contribuyentes, llevándose consigo la eliminación a auditoría financiera la cual es el punto de partida para dictamen fiscal, hay que recordar que las normas de información financiera constituyen la normatividad base para el cálculo de los impuestos federales en México. Es importante señalar que por decreto presidencial publicado el 30 de junio de 2010 , se estableció una facilidad administrativa para, cumpliendo con cierta información el contribuyente obligado a dictaminar optara por no hacerlo (Decreto, 2010). La eliminación de la obligatoriedad del dictamen, a criterio del SAT, parte del supuesto de la poca recaudación que el mismo efectuaba, no obstante haber recaudado en promedio del 2005 al $2010 \$ 17,910$ millones de pesos a través de este instrumento de fiscalización (IMCP, 2013). El hecho de dictaminarse para efectos fiscales si bien es cierto trae consigo

\section{La auditoría de estados financieros}


un costo que se traslada al contribuyente, aunado al hecho el tiempo que se invierte internamente en la compañía para atender la revisión, también trae consigo ventajas adicionales tales como tener como medida de control interno a especialistas que revisen tanto la información financiera como la información fiscal de los contribuyentes de manera oportuna, de tal forma que si detectan desviaciones a las disposiciones fiscales puedan autocorregirse y al hacerlo de forma espontánea de conformidad al artículo 33 del CFF no procederían las multas (CFF, 2018), por otro lado, también existe la posibilidad de que detecten áreas de mejora, pues pueden existir también errores involuntarios que repercutan en el pago de mayores impuestos a los debidos; La posibilidad de que el auditor detecte estas omisiones es más alta que la del propio contribuyente auditado en virtud a que este último no tiene las misma experiencia y capacitación que el auditor, adicionalmente no cuenta con suficiente personal fiscal para atender la propia operación fiscal de su negocio (Hernández, 2011). Otra ventaja directa de dictaminarse es el hecho de que la autoridad está obligada, de conformidad al artículo 52-A del CFF, con sus excepciones, a iniciar sus facultades de comprobación primeramente con el auditor y no con el contribuyente, eso ayudaría a que un especialista atendiera las observaciones que tenga el SAT en el ejercicio de sus facultades de comprobación. Otra ventaja adicional es la conclusión anticipada de visitas domiciliarias del SAT cuando te dictaminas fiscalmente, de conformidad al artículo 47 del CFF lo que sin duda alguna dará tiempo adicional al contribuyente, pues se presume su veracidad con fundamento en el artículo 52 del CFF. Adicionalmente, al dictaminarse fiscalmente y el auditor determine diferencias de impuestos a las

Hernández, A., Martínez, V., Luna A. \& Hernández J. 
calculadas por el contribuyente, se tiene un plazo de 10 días hábiles para enterar las diferencias a cargo considerándose el pago de manera espontaneo, y se tiene por cumplida la presentación de la DISIF, según el artículo 32-A del CFF. Por otro lado, el contribuyente de manera indirecta al dictaminarse, proporciona a todos los usuarios de su información financiera y fiscal la confianza de que la información esta revisada por un experto, esto es de utilidad para la propia compañía pues esto contribuye reducir posibles contingencias fiscales, proporciona una imagen de credibilidad y responsabilidad tributaria, para los trabajadores constituye una ventaja de seguridad financiera de la empresa que representa y sobre todo en materia de la participación de los trabajadores en las utilidades de las empresas a las que tiene derecho de conformidad con el artículo 123 apartado A fracción IX de la CPEUM (CPEUM, 1917) y al título tercero, capítulo VII de la Ley Federal de Trabajo (LFT).

En adición a lo anterior, y tomando en cuenta que en México el $99.8 \%$ de las empresas son pequeñas y medianas (Arana, 2018), difícilmente cumplirían con el supuesto de optar por realizar una auditoría financiera para efectos fiscales, por lo tanto difícilmente sus Estados Financieros serían revisados por un auditor independiente, por lo que de no tener controles fiscales adecuados podrían estar expuestos a incumplimientos fiscales lo que implicaría que el efectuar operaciones comerciales con ellas o entre ellas podría estar en riesgo de llevarse a cabo, adicionalmente el considerar dictaminarse financieramente, lo que implícitamente se lleva a cabo en una auditoría fiscal, puede representar una ventajas adicionales, por citar una, según estudio realizado en el Reino Unido, las pequeñas empresas obtuvieron mayor calificación

\section{La auditoría de estados financieros}


crediticia cuando elegían dictaminarse voluntariamente respecto a las que no lo hacían (Dedman \& Kausar, 2012). Los Contadores Públicos Inscritos (CPIS) que emitieron Dictamen Fiscal (SAT1, 2018) se refleja en la figura 3:

Figura 3. Relación de CPIS que emitieron Dictamen Fiscal por año

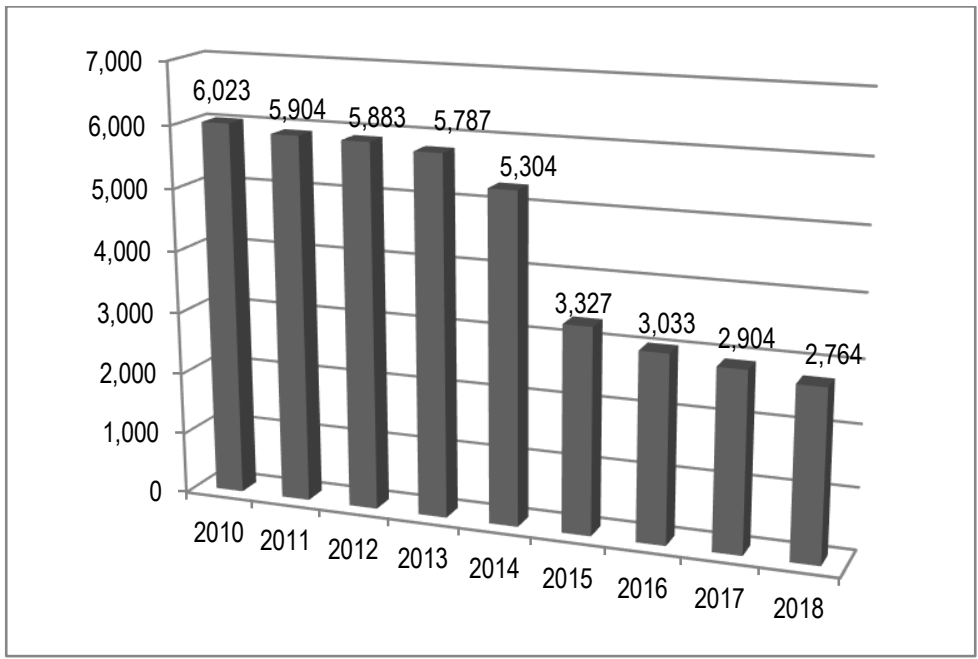

Fuente: Elaboración propia con datos del SAT a octubre de 2018

De la figura anterior se desprende que aún y cuando existía alternativa para evitar la obligación de dictaminarse, la cantidad de dictaminadores se mantuvo prácticamente de 2010 a 2013; en 2014 sufrió una ligera disminución, pero a partir de ese año disminuyó más del $50 \%$ respecto al año de 2013.

Hernández, A., Martínez, V., Luna A. \& Hernández J. 


\section{Metodología}

Se analizó la información financiera y fiscal relativa a las empresas 0 grupos de ellas que se encuentran registradas como emisoras de acciones en la BMV en el año de 2018, para efectos de determinar la población objetivo se separaron aquellas que están domiciliadas en Nuevo León las cuales son objeto de estudio (Grupos emisores con domicilio en NL). Una vez identificados los grupos de empresas emisoras se revisaron las notas a los Estados Financieros para efectos de identificar aquéllos que seguían dictaminándose voluntariamente, una vez identificados se comprobó con los auditores (Big Four) que prestaban esos servicios; posteriormente se aplicaron encuestas a expertos trabajadores de los grupos con puesto de Gerente o Director fiscal para determinar la razón del porque continuaban dictaminándose para efectos fiscales, el $60 \%$ de los grupos emisores respondió la encuesta. Paralelamente y con base a la teoría de la concentración de los servicios de auditoría (Toscano, 2014), se aplicaron encuestas a expertos gerentes y socios las Big Four ubicadas en NL quienes son las que dictaminan los grupos emisores de acciones, se obtuvo respuesta del $100 \%$ de dichas firmas; a la información obtenida de ambas encuestas se aplicó la metodología del expertones por lógica borrosa la cual consiste en la ponderación de pesos acumulados (Hernández, 2011), para efectos de

\section{La auditoría de estados financieros}


identificar las principales cusas de que sigan aplicando el Dictamen Fiscal.

\section{Resultados}

En relación a las encuestas aplicadas a los grupos de empresas de la BMV ubicadas en NL, se encontró que, con un peso del 0.900 , se mantiene el dictamen fiscal para disminuir sus contingencias fiscales. Por otro lado se encontró que los medios electrónicos fueron la principal causa del porque el SAT eliminó e dictamen fiscal con un peso de 0.900, seguido por que las expectativas del SAT no fueron cumplidas con un peso de 0.857 y por las pocas omisiones de impuestos detectadas. Por otro lado, la principal ventaja del dictamen fiscal es el que el mismo se mantiene como una medida de control interno fiscal con un peso del 0.829 .

Con respecto a las encuestas aplicadas a las Big Four se encontró que con un peso del 0.780 se mantiene el dictamen fiscal para disminuir sus contingencias fiscales, este resultado es coincidente con los resultados de las encuestas aplicadas los grupos emisores. Por otro lado se encontró que los medios electrónicos fueron la principal causa del porque el SAT eliminó e dictamen fiscal con un peso de 0.930, este resultado coincide como la variable de mayor peso al igual que la encuesta aplicada a las emisoras, seguida por las expectativas del SAT no fueron cumplidas con un peso de 0.690; cabe señalar que otra variable de peso considerable fue el alcance en la revisión del auditor con un peso de 0.680 , es decir, el auditor en principio considera que debió haber dado

Hernández, A., Martínez, V., Luna A. \& Hernández J. 
mayor alcance a sus pruebas de auditoría. Por otro lado, la principal ventaja del dictamen fiscal es el que el mismo se mantiene como una medida de control interno fiscal con un peso del 0.710; este resultado, aunque con menor peso, también es coincidente con los resultados de las encuestas aplicadas a los grupos emisores.

\section{Conclusiones}

La reforma fiscal 2014 trajo consigo la eliminación de la mayor parte de los dictámenes fiscales e inherentemente trajo consigo la desaparición de la misma cantidad de auditorías financieras y con ello la validación de la información financiera por un auditor externo certificado, lo que afectó a los usuarios de dicha información, entre ellos, sus proveedores o clientes, la misma autoridad, etc., pues el dictamen constituía una garantía adicional de que las operaciones con los terceros se realizarían pues tenía la certificación de un auditor externo que emitía una opinión sobre la razonabilidad de los estados financieros auditados, lo que representaba al menos que el negocio podría continuar operando. En adición a lo anterior, al no ejercer la opción de dictaminarse fiscalmente, puede afectar a los propios contribuyentes que no cuenten con estructura fiscal suficiente $o$ con los controles internos necesarios para de manera preventiva evitar incurrir en omisiones de impuestos.

Desde la perspectiva de los trabajadores, respecto a las empresas que anteriormente se dictaminaban, contaban al menos con el hecho de que los resultados financieros y fiscales de las empresas eran revisados por un CPR independiente, eso fortalecía la credibilidad en la información sobre todo para efectos de la PTU, situación que quedó sin

\section{La auditoría de estados financieros}


esa revisión, a menos que los propios trabajadores soliciten una revisión ante el SAT.

Respecto a las empresas y a los auditores que las dictaminan, coinciden en el hecho de que el dictamen fiscal disminuye el riesgo de incurrir en contingencias fiscales y constituye un instrumento de control interno fiscal preventivo, es por ello que aún y cuando ya no es obligatoria su adopción, siguen conservándolo. Lo anterior muy relevante, pues las autoridades tributarias han comenzado su fiscalización apoyada fuertemente en las tecnologías de información, por lo tanto su revisión, además de ser eficiente y eficaz, descansa en la propia información que el contribuyente proporciona a través de sus CFDI, declaraciones electrónicas, contabilidad electrónica, etc., por tanto se vuelve relevante el establecimiento de controles internos fiscales que puedan soportar con éxito cualquier revisión electrónica por parte de las autoridades fiscales. El hecho de contar con dictamen fiscal, constituye una verdadera herramienta de control pues representa al menos el hecho de que personal capacitado valida la información financiera y fiscal; sin embargo se limitó su utilización al quedar este de manera opcional y solo para ciertos contribuyentes; por lo que es importante que se evalúe su resurgimiento ampliando la gama de contribuyentes que deseen utilizarlo, pues aunque para muchos suele representar un gasto, la evidencia demuestra que el mismo sigue siendo utilizado por los grandes grupos para evitar contingencias fiscales, por lo que es muy probable que las PYMES se encuentren más desprotegidas en materia de control interno fiscal y personal fiscal, al contar con menores recursos destinados a ello.

Hernández, A., Martínez, V., Luna A. \& Hernández J. 


\section{Referencias}

Arana, D. (2018). Pymes mexicanas, un panorama para 2018. Forbes México. Disponible en: https://www.forbes.com.mx/pymes-mexicanas-un-panoramapara-2018/

Barquero, M. (2008). El control interno en trabajos de fiscalización. Auditoría y Gestión de los Fondos Públicos (47), p. 48. Disponible en: http://asocex.es/wpcontent/uploads/PDF/200904_47_47.pdf

Carpi, C. (2010). Práctica de auditoría automatizada. Instituto Mexicano de Contadores Públicos. México

Centro de Estudios de Finanzas Públicas (2019). Paquete Económico 2019. Disponible en: http://www.cefp.gob.mx/new/paquete_economico_2019i.php

Chan, W., Mauborgne R. (2005). Blue ocean strategy: How to create uncontested market space and make the competition irrelevant. Long Range Planning, 41(2). 226228. doi: 10.1016/j.Irp.2008.02.003

Código de Comercio (2018). Diario Oficial de la Federación, México, 28 de marzo de 2018. Disponible en: http://www.diputados.gob.mx/LeyesBiblio/ref/ccom.htm

Código Fiscal de la Federación (2014). Diario Oficial de la Federación. México, 9 de diciembre de 2013.2 Disponible en: http://dof.gob.mx/nota_detalle.php?codigo=5324942\&fecha=09/12/2013

Código Fiscal de la Federación, (2018). Diario Oficial de la Federación. México, 25 de junio de 2018.2 Disponible en: https://www.dof.gob.mx/nota_detalle.php?codigo=5528960\&fecha=25/06/201 8

Consejo Mexicano de Normas de Información Financiera (2018). Normas de Información financiera. Disponible en: http://imcp.org.mx/servicios/las-normasinformacion-financiera/

Constitución Política de los Estados Unidos Mexicanos (2018). Disponible en: https://www.juridicas.unam.mx/legislacion/ordenamiento/constitucion-politicade-los-estados-unidos-mexicanos

\section{La auditoría de estados financieros}


84

Decreto, (2010). Decreto por el que se otorgan facilidades administrativas en material de simplificación tributaria publicado el 30 de junio de 2010. Disponible en: http://dof.gob.mx/nota_detalle.php?codigo=5149836\&fecha=30/06/2010

Dedman E. \& Kausar A., (2012) The impact of voluntary audit on credit ratings: evidence from UK private firms. Accounting and Business Research, 42(4), 397-418. doi: 10.1080/00014788.2012.653761

Diario Oficial de la Federación (2017). Resolución Miscelánea Fiscal, Anexo 5, 16 de mayo de 2017.2 Disponible en: http://www.dof.gob.mx/nota_detalle.php?codigo=5482871\&fecha=16/05/2017

Diario Oficial de la Federación (2018). Ley de Impuesto Sobre la Renta, 18 de noviembre de 2018.2 Disponible en: http://www.diputados.gob.mx/LeyesBiblio/pdf/LISR_301116.pdf

Diario Oficial de la Federación (2018). Ley de Ingresos de la Federación, 15 de noviembre de 2017.2 Disponible en: http://dof.gob.mx/nota_detalle.php?codigo=5504645\&fecha=15/11/2017

Diario Oficial de la Federación (2018). Ley del Mercado de Valores, 30 de diciembre de 2005. Disponible en: http://www.diputados.gob.mx/LeyesBiblio/ref//mv.htm

Diario Oficial de la Federación (2018). Ley Federal del Trabajo, 22 de junio de 2018. Disponible en: http://www.diputados.gob.mx/LeyesBiblio/ref/Itt.htm

Diario Oficial de la Federación (2018). Ley General de Sociedades Mercantiles, 14 de junio de 2018.2 Disponible en: http://www.diputados.gob.mx/LeyesBiblio/ref/lgsm.htm

Diario Oficial de la Federación (2018). Resolución Miscelánea Fiscal, 28 de mayo de 2018.

Disponible en: http://omawww.sat.gob.mx/fichas_tematicas/dictamen_fiscal_info_alternatival Paginas/articulo_32_calendario.aspx

Ernst \& Young Global Limited (2013).Código Fiscal de la Federación. Disponible en: http://www.eyboletin.com.mx/eysite2/index.php?option=com_content\&task=vi ew\&id $=1632 \& b=1$

Fayol, H. (1961). Administración Industrial y General. México: Herreo Hermanos, S. A. Disponible en: http://maryperez.galeon.com/admonindustrial.pdf

Hernández, A., Martínez, V., Luna A. \& Hernández J. 
Fernández, F., Vera, J., Silvia. (2015). La mala praxis en el mundo corporativo: el caso Enron. Universidad Pontificia Comillas. Madrid. Disponible en: http://hdl.handle.net/11531/3971

Hay, D., (2002). The Voluntary Choice of an Audit of Any Level of Quality. Auditing a journal of practice and theory, 23(2). 37-54 Disponible en: https://www.researchgate.net/publication/236737238_The_Voluntary_Choice _of_an_Auditor_of_Any_Level_of_Quality

Hernández, A. (2011). Análisis crítico del control interno fiscal y su incidencia en las contingencias fiscales en la industria manufacturera de Nuevo León (Tésis doctoral). Universidad Autónoma de Nuevo León Facultad de Contaduría Pública y Administración. Nuevo León, México. Disponible en: http://eprints.uanl.mx/2469/

Hernández, A. (2016). El control interno contable y fiscal como medida para contribuir a la maximización de los resultados financieros de los negocios. Innovaciones de Negocios, 13(25). 47-69. Disponible en: http://eprints.uanl.mx/12630/1/13.25\%20A3.pdf

Hernández, J. (2016). Aplicación tecnológica en los impuestos. PWC, Webcast. Disponible en: https://www.youtube.com/watch?v=03DSDYsPC6E

International Auditing and Assurance Standards Board (2009). Norma internacional de auditoría 320: Importancia Relativa o Materialidad en la Planificación y Ejecución de la Auditoría. Disponible en: http://www.icac.meh.es/NIAS/NIA\%20320\%20p\%20def.pdf

International Financial Reporting Standards (2018). About us. Estados Unidos Disponible en: http://www.ifrs.org/About-us/Pages//FRS-Foundation-andIASB.aspx

Koontz, H. y Weihrich, (1994). Administración una perspectiva global. México: McGraw Hill

Largay, J (2002). Lessons from Enron. Accounting Horizons, 16(2). 153-156. Disponible: http://dx.doi.org/10.2308/acch.2002.16.2.153

Mears, B (2005). Arthur Andersen conviction overturned. CNN International. Disponible en: http://edition.cnn.com/2005/LAW/05/31/scotus.arthur.andersen/

\section{La auditoría de estados financieros}


Ojala, H., Collis, J., Kinnunen, J., Niemi, L., Troberg, P. (2014). Why do owner-managers of micro-companies opt for voluntary audits? The importance of debt providers' versus entrepreneurs' needs. SSRN Electronic Journal. doi: $140.2139 /$ ssrn. 2617169

Price Waterhouse Coopers, (2014).Punto de Vista. 2014, año de transición al nuevo coso 2013. Disponible en: https://studylib.es/doc/4935080/2014--a\%C3\%B10de-transici\%C3\%B3n-al-nuevo-coso-2013

Robbins, S., y Coulter, M. (2000). Administración. México: Prentice Hall

Servicio de Administración Tributaria (2018). Pagina Oficial del SAT, 25 de noviembre de 2018. Disponible en: http://omawww.sat.gob.mx/transparencia/Documents/TTG\%202do\%20trimest re\%202018_180828.pdf

Servicio de Administración Tributaria, (2018). Pagina Oficial del SAT, 25 de noviembre de 2018. Disponible en: https://datos.gob.mx/busca/dataset/padron-decontribuyentes

Toscano, J., Álvarez, C., Montano, C., García, M., (2014). El poder de mercado y la concentración económica en los servicios de auditoría. Revista Universo Contábil, Enero-Marzo, 145-165

United States Securities and Exchange Comission (2018). Home. Disponible en: http://www.sec.gov

United States Securities and Exchange Commission (2002). Sarbanes-Oxley Law. Disponible en: https://www.sec.gov/spotlight/sarbanes-oxley.htm

Hernández, A., Martínez, V., Luna A. \& Hernández J. 